\title{
An Unusual Bilateral Duplication of the Suprascapular Vein and Its Relation to the Superior Transverse Scapular Ligament Revealed by Anatomage Table
}

\author{
Eleni Panagouli, Alexandra Tsirigoti, Georgia Kotsira, Theano Demesticha, Panagiotis \\ Skandalakis, Theodore Troupis, Dimitrios Filippou
}

Department of Anatomy and Surgical Anatomy, Medical School, National and Kapodistrian

University of Athens

Correspondence:

eleni72000@yahoo.gr

Tel.: + 302107462394

Fax.: + 302107462398

Received: 21 July 2019; Accepted: 28 December 2019

\begin{abstract}
Objective. The aim of our paper is to present a rare variation of the suprascapular vein, its incidence and clinical significance. Case Report. A rare case of a double suprascapular vein was observed in a digitalized human cadaver on Anatomage Table 5.0. The vein divided into two branches, one passing over the transverse scapular ligament, while the other one coursed underneath the ligament, inside the notch. Conclusion. This variation has major clinical importance as it is associated with the appearance of Suprascapular nerve entrapment syndrome.
\end{abstract}

Key Words: Suprascapular Artery • Scapular Notch • Suprascapular Nerve - Transverse Scapular Ligament 3D Anatomy.

\section{Introduction}

In most cases, the Suprascapular vein (SV) passes through the base of the posterior triangle of the neck, and drains into the external jugular, which is a branch of the subclavian vein (1). In the middle half of the superior border of the scapula, the SV is usually accompanied by the suprascapular artery (SA). Most commonly, both the artery and the vein pass above the superior transverse scapular ligament (STSL), while the suprascapular nerve (SN) passes through the foramen formed by the STSL $(1,2)$. The suprascapular nerve derives from the superior trunk of the brachial plexus, passes through the suprascapular notch, under the STSL and ends at the infraspinatus fossa (3). According to the literature, the location of the SA, SN and SV seems to be highly variable, presenting different patterns (3), with multiple veins being reported in $21.3 \%$ of cases (4).

The aim of our paper is to present an interesting clinically significant variation of the SV which to our knowledge has only been presented in one study in the available literature (3). Additionally, the novelty of the case is that it was revealed in a digitalized human cadaver on Anatomage Table 5.0.

\section{Case Report}

An electronic anatomical review was performed on digital cadavers included in Anatomage Table 5.0. Anatomage Table 5.0 is an advanced $3 \mathrm{D}$ anatomy visualization 


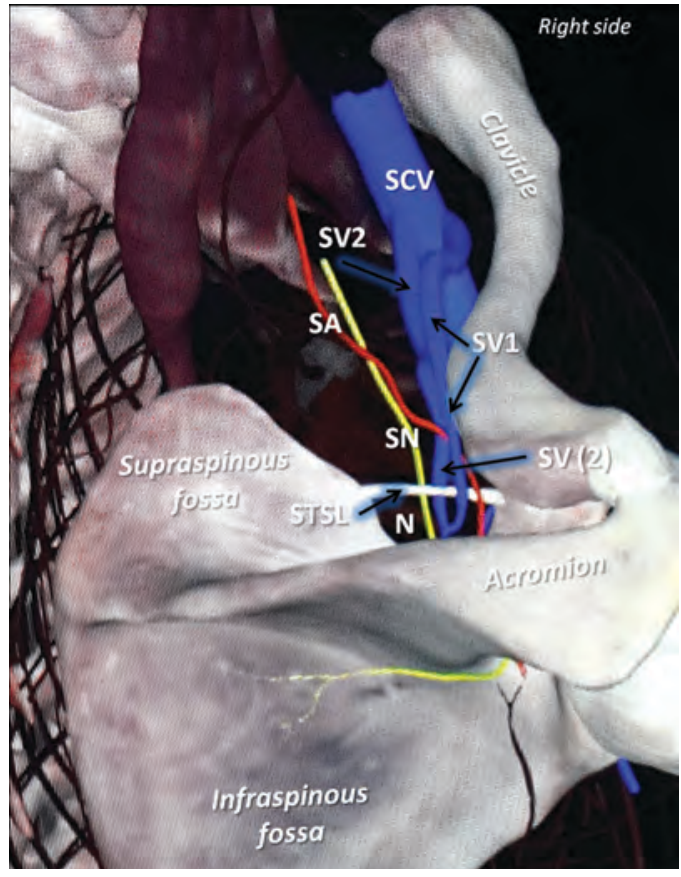

Figure 1. Right side: The suprascapular vein (SV) divided into two branches, one (SV1) passing over the superior transverse scapular ligament (STSL) with the suprascapular artery (SA), and the other (SV2) below the ligament, inside the notch $(\mathrm{N})$, together with the suprascapular nerve (SN). Both of them drain into the subclavian vein (SCV). Figures are published with the permission of Anatomage Inc. system developed for educational purposes, which has been adopted by many of the world's leading medical schools and institutions. According to the description of the product and its creators, Anatomage Table 5.0 is the only fully segmented real human $3 \mathrm{D}$ anatomy system. Users are able to visualize anatomy exactly as they would on a fresh cadaver. Individual structures are reconstructed in accurate $3 \mathrm{D}$, resulting in an unprecedented level of real accurate anatomy, dissectible in 3D. Anatomy is presented as a fully interactive, life-sized touch screen experience. The Anatomage Table 5.0 contains three digitalized cadavers, specifically a Korean female who died of pneumonia (gastric cancer), a Caucasian male sentenced to death and diagnosed with a brain tumor, and a Korean male who died from respiratory complications, while being treated for acute leukemia.

In the present case, we discovered a bilateral case of a double SV in the Korean female cadaver, who contracted her disease at the age of 26. The SV was divided in two branches on both sides, one passing over the

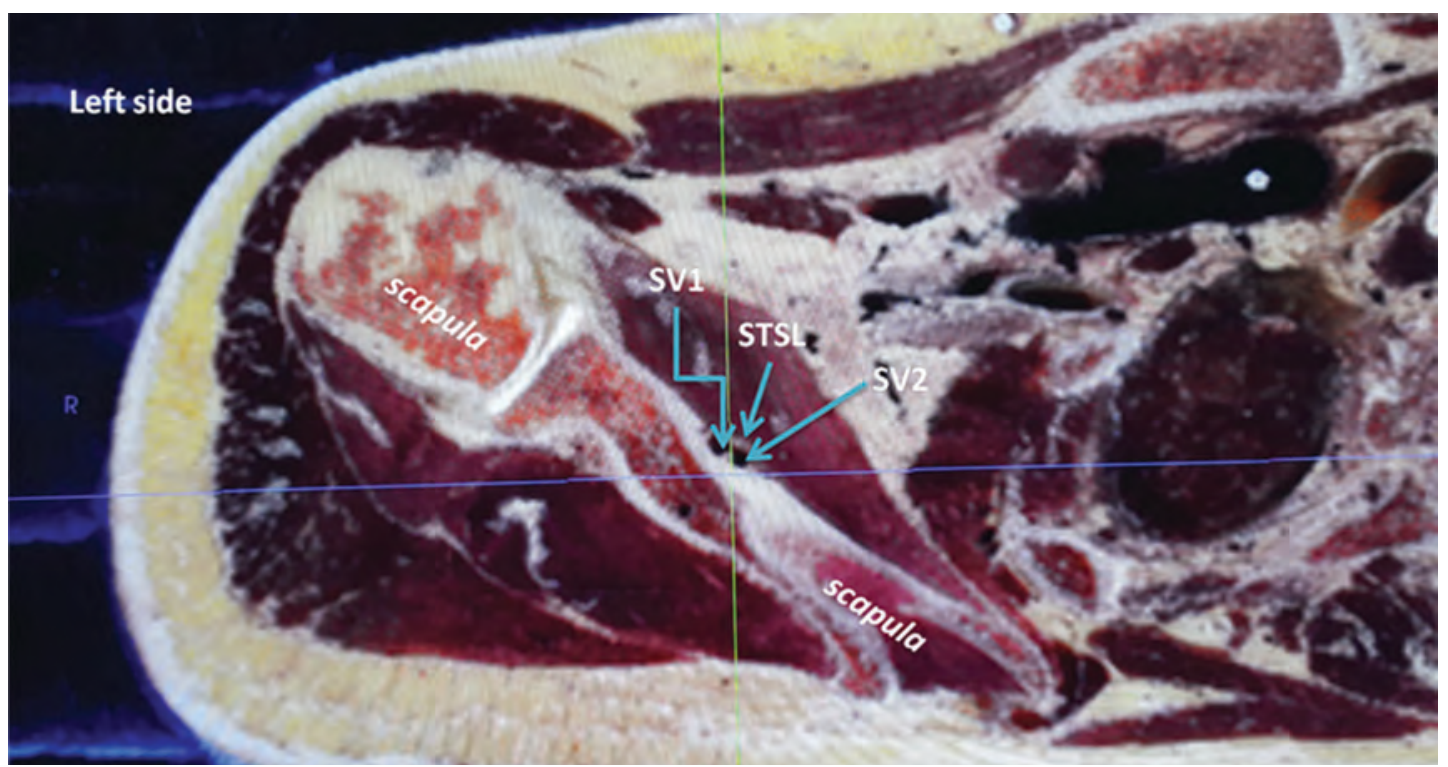

Figure 2. Left side: Cross section - Double suprascapular vein (SV), the one (SV1) passing over the superior transverse scapular ligament (STSL) and the other one (SV2) inside the notch. Figures are published with the permission of Anatomage Inc. 
STSL, together with the suprascapular artery, while the other one coursed underneath the ligament, inside the notch, accompanying the SN (Figure 1,2). The two branches drained independently into the subclavian vein, one at the anterior and the other at the posterior aspect of the vein (Figure 1). The SA and nerve followed the typical course on both sides. We did not observe other artery or vein variations in the shoulder region.

\section{Discussion}

The SV presents major deviations in its course and number, according to the available literature. The vein may be duplicated in some cases $(2.4 \%-19.8 \%)$ and triplicated in others (1.98\%) and it might pass above or below the STSL (variable incidence) $(3,4)$. Two major studies have been conducted on the SV's pattern $(3,4)$. Yang et al. in their study performed an anatomical examination of 103 cadaveric shoulders from 55 Korean cadavers (53 right shoulders, 50 left shoulders including 28 males and 27 females; mean age, 70.4 years; range, 36-94 years) (3). In all cases, a single artery and nerve were mentioned, whereas the number of the SVs varied, $19.4 \%$ of them being double and $1.9 \%$ triple (3). Apart from two rare cases in which the ligament or the ossified bridge was absent (thus a total of 101 STSLs were studied), in all the other cases the $\mathrm{SN}$ passed under the STSL (101/103, 95.4\% of the shoulders). The arrangement of the vessels in the suprascapular notch varied (Table 1 ).

Yang et al. classified the location of the suprascapular vessel into three types: Type I (59.4\% of the cases) where all suprascapular vessels pass above the STSL, Type II where the vessels cross over and under the STSL (29.7\%) and Type III (10.9\%) where the suprascapular vessels pass below the suprascapular ligament or bony bridge (3). In Type II four subtypes were mentioned. Our case corresponds to subtype IIa (10/101 shoulders, estimated according to Yang et al. up to 9.9\%) where the SA runs above the STSL and the SV is divided, with the two branches crossing simultaneously above and below the ligament.

Polguj et al. presented a new classification of the suprascapular vessels at the suprascapular notch region, after examining 106 formalin-fixed cadaveric shoulders (55 right, 51 left) (4). In all the shoulders (100\%,

Table 1. Classification of the Course of Suprascapular Vessels

\begin{tabular}{lll}
\hline Topography of suprascapular vessels around STSL & $\begin{array}{l}{ }^{*} \text { Cadavers=101; } \\
(\% ; \mathrm{N})\end{array}$ & $\begin{array}{l}{ }^{+} \text {Cadavers=106; } \\
(\% ; \mathrm{N})\end{array}$ \\
\hline All vessels above STSL & $54.4(55)$ & (I) 17 (18) \\
\hline All vessels below STSL & $9.9(10)$ & (II) $12.3(13)$ \\
\hline Artery above and vein below STSL & $2.9(3)$ & (III) $61.3(65)$ \\
\hline Artery below and vein above STSL & $10.9(11)$ & - \\
\hline Artery above and double vein adversarially STSL & $9.9(10)$ & - \\
\hline Artery below and double vein adversarially STSL & $3.9(4)$ & (IV) 0.94 (1) \\
\hline Artery below and double vein below STSL & $0.99(1)$ & - \\
\hline Artery and double vein above STSL & $4.95(5)$ & (IV) 8.5 (9) \\
\hline Artery and two branches of triple vein above STSL & $1.98(2)$ & \\
and one branch below STSL & - & \\
\hline Other variations ${ }^{\ddagger}$ & &
\end{tabular}

"Yang et al. (3); ${ }^{\text {P}}$ Polguj et al. (4); STSL=Superior transverse scapular ligament; ${ }^{*}$ Other variations refer to accessory veins cases in which the vessels passed under the anterior coracoscapular ligament. 
106 out of 106 shoulders) the SN passed below the STSL. Polguj et al. classified the topography of the suprascapular vessels with reference to STSL in four types (Table 1) (4). Type IV included only two extremities (2.4\%) with double SV. On the first, both branches of the double SV ran above the STSL and on the second beneath the anterior coracoscapular ligament. None of these types corresponded to our case.

The course and location of the suprascapular vessels are of major clinical significance. Suprascapular nerve entrapment syndrome (SNES) is a neuropathy caused by the compression of the $\mathrm{SN}$ during its course through the suprascapular notch. SNES was first described by André Thomas in 1936 and is responsible for pain and dysfunction of the shoulder girdle at a rate of $1-2 \%$ (5). This syndrome affects the infraspiratus and supraspiratus muscles, which are innervated from the $\mathrm{SN}$, causing their atrophy.

According to Labertowicz et al. the occurrence of an accessory vein (double or triple vein), in cases in which the SA crosses under the STSL together with the SN, resulted in nerve damage due to blood pressure and high incidence of SNES (6). Other causes of nerve damage include traumatic injuries in the areas of the clavicle or scapula and its joints, iatrogenic injuries in the process of a surgical operation, excessive overload in athletes or physical laborers, tuberous alterations of this area, or even systemic diseases.

\section{Conclusion}

The presence of a double SV is mentioned in only two articles $(19.8 \%-20 / 101$ cases in the article of Yang et al., 2.4\%, 2/106 cases in the article of Polguj et al.) according to the international available bibliography. Specifically, a double SV passing adversarial to the STSL, as presented in our case, was reported only in the research by Yang et al. (Type IIa
- 10/101, 9.9\%). This variation has major clinical importance as the arrangement and number of the vessels crossing through the foramen is associated with the appearance of SNES. Additionally, knowledge of the Suprascapular triad's analytic topography may prevent surgical injuries during procedures.

\section{What Is Already Known on this Topic:}

The Suprascapular vein (SV), in most cases, passes above the superior transverse scapular ligament (STSL) accompanied by the suprascapular artery (SA), while the SN passes through the foramen, formed by the STSL. Variations of the course of the $S V$ are not rare and present important clinical significance, especially for Suprascapular nerveentrapment syndrome (SNES).

\section{What this Case Adds:}

We present a bilateral double SV, one passing over the STSL together with the suprascapular artery, while the other one courses underneath the ligament with the SN. Such an arrangement is rare, found in about $9.9 \%$ of cases. This variation has major clinical importance as the arrangement and number of vessels crossing through the foramen is associated with the appearance of SNES. Moreover, the novelty of our paper is that this variation was observed in a digitalized cadaver on Anatomage Table 5.0, the only fully segmented real human 3D anatomy system.

Authors' Contributions: Conception and design: EP and DF; Acquisition, analysis and interpretation of data: AT, GK and TD; Drafting the article EP, AT and GK; Revising it critically for important intellectual content: TT, DF and SP; Approved final version of the manuscript: EP, AT, GK, TD, TT, PS and DF.

Conflict of Interest: The authors declare that they have no conflict of interest.

\section{References}

1. Standring S, Borley NR, Collins P, Crossman AR, Gatzoulis MA, Healy JC, et al. (editors.). Gray's anatomy. The anatomical basis of clinical practice. 40th ed. Edinburgh: Elsevier; 2008.

2. Pyrgakis P, Panagouli E, Venieratos D. Anomalous Origin and Course of the Suprascapular Artery Combined with Absence of the Suprascapular Vein: Case Study and Clinical Implications. N Am J Med Sci. 2013; 5(2):129-33.

3. Yang HJ, Gil YC, Jin JD, Ahn SV, Lee HY. Topographical anatomy of the suprascapular nerve and vessels at the suprascapular notch. Clin Anat. 2012;25(3):359-65. 
4. Polguj M, Rozniecki J, Sibiński M, Grzegorzewski A, Majos A, Topol M. The variable morphology of suprascapular nerve and vessels at suprascapular notch: a proposal for classification and its potential clinical implications. Knee Surg Sports Traumatol Arthrosc. 2015;23(5):1542-8.

5. Pećina M. Who really first described and explained the suprascapular nerve entrapment syndrome? J Bone Joint Surg Am. 2001;83(8):1273-4.
6. Labętowicz P, Synder M, Wojciechowski M, Orczyk K, Jezierski H, Topol M, et al. Protective and Predisposing Morphological Factors in Suprascapular Nerve Entrapment Syndrome: A Fundamental Review Based on Recent Observations. Biomed Res Int. 2017;2017:4659761. 